\title{
Estimation Characteristics of Input Voltage Waveform of Single-Phase PFC Converter for Both Sinusoidal and Rectangular Input Voltages
}

\author{
Hiroatsu Fukuoka Member (Nagoya Institute of Technology) \\ Quan Li Non-member (Nagoya Institute of Technology) \\ Toshiyuki Kouno Student Member (Nagoya Institute of Technology) \\ Takaharu Takeshita Member (Nagoya Institute of Technology)
}

Keywords: single-phase PFC converter, rectangular-waveform distribution system, estimation of input voltage

A lot of industrial equipment and household electric appliances have diode rectifier circuits for the dc voltage source of the inverter circuits. However, the input current waveform of the diode rectifier circuit is distorted. To avoid this problem, PFC converter have attracted considerable attention. However, the system configuration is complication compared with that of the diode rectifier circuit.

On the other hand, the single-phase or three-phase distribution system with the rectangular voltage waveform has been proposed. The distribution system with the rectangular voltage waveform is useful for the equipment with the dc voltage source because the high input power factor is obtained by using the diode rectifier circuits whose system configuration is simple.

This paper presents a single-phase PFC converter for both the distribution systems with the sinusoidal or rectangular voltage waveform. Also, this paper presents an input voltage estimation because it is necessary to judge between the sinusoidal and rectangular voltage waveforms and to obtain the high input power factor.

Figure 1 shows the rectangular input voltage waveform. The function $\operatorname{rec} \theta$ is defined as the rectangular waveform of which the

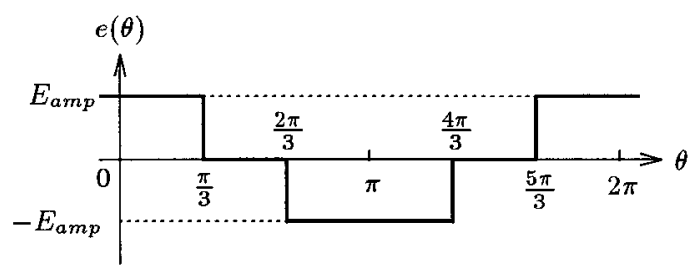

Fig. 1. Rectangular input voltage waveform

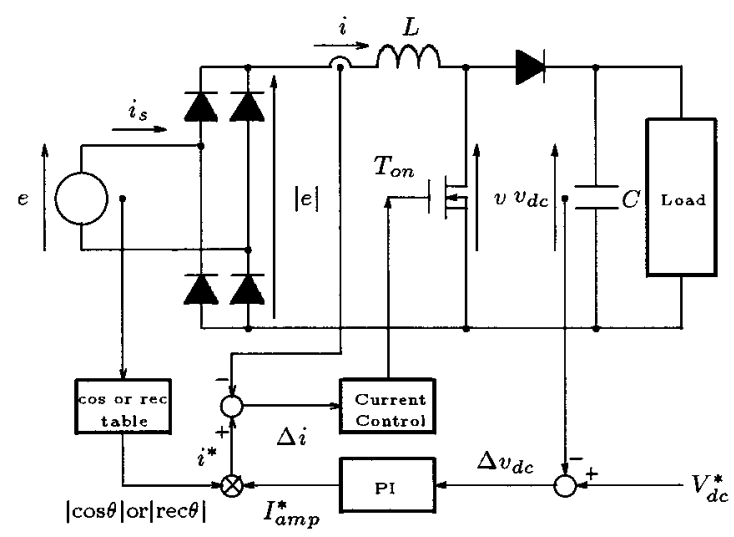

Fig. 2. System configuration of single-phase PFC converter amplitude $E_{\text {amp }}$ equals 1 .

Figure 2 shows the system configuration of the single-phase PFC converter for both sinusoidal and rectangular input voltage waveforms. The control purpose of PFC converter is the realization of the unity effective power factor and the stable output dc voltage.

The amplitude $E_{R M}(n)$ and the phase angle $\theta_{R M}(n)$ of the rectangular input voltage can be estimated with the estimation error of the input voltage $\Delta e_{R}(n-1)$ in (1) and (2)

$$
\begin{aligned}
& E_{R M}(n)=E_{R M}(n-1)+K_{R E} \Delta e_{R}(n-1) \operatorname{rec} \theta_{R M} \ldots \ldots \ldots(1) \\
& \theta_{R M}(n)=\theta_{R M}(n-1)+\omega_{R} T_{c}-K_{R \theta} \Delta e_{R}(n-1) \operatorname{rec}\left(\theta_{R M}-\pi / 2\right)
\end{aligned}
$$

where $K_{R E}$ and $K_{R \theta}$ are the gains for the estimation of the amplitude and the phase angle, respectively. $\omega_{R}$ is the angular frequency of the input voltage and $T_{c}$ is the control period.

Figure 3 shows the convergence characteristics for the phase angle disturbances of $-180 \sim+180 \mathrm{deg}$.

Using the proposed scheme, the input voltage estimation algorithm can converge the estimation errors within $40 \mathrm{~ms}$ of the input voltage for the amplitude and phase angle disturbances. The power factors are 0.995 and 0.949 when the sinusoidal and rectangular waveforms are used as the input voltages, respectively.

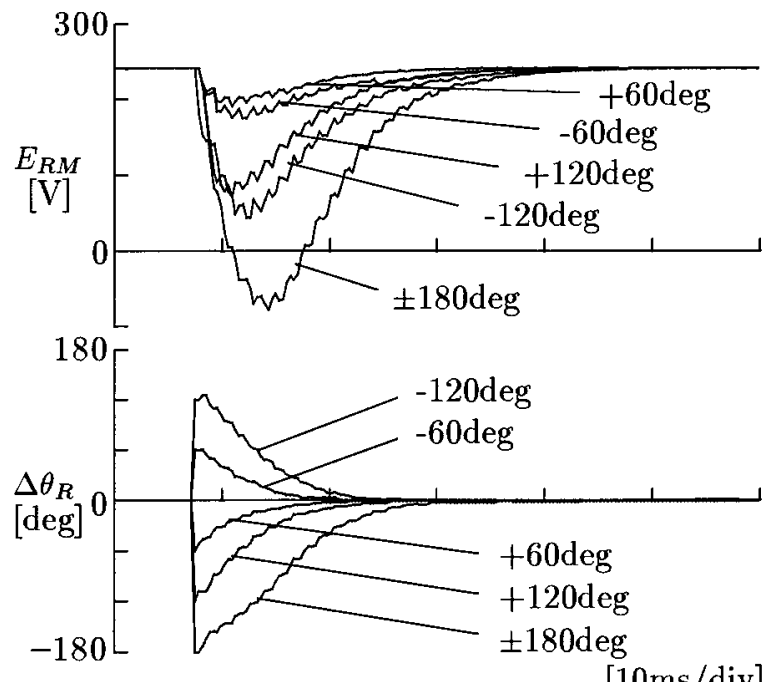

$[10 \mathrm{~ms} / \mathrm{div}]$

Fig. 3. Convergence characteristics for phase angle disturbances 


\title{
Estimation Characteristics of Input Voltage Waveform of Single-Phase PFC Converter for Both Sinusoidal and Rectangular Input Voltages
}

\author{
Hiroatsu Fukuoka* Member \\ Quan $\mathrm{Li}^{*} \quad$ Non-member \\ Toshiyuki Kouno* Student Member \\ Takaharu Takeshita* Member
}

\begin{abstract}
The distribution system with the rectangular voltage waveform is useful for the electrical equipment with the dc voltage source because the high input power factor is obtained by using the diode rectifier circuits. This paper presents a single-phase type AC/DC converter using a novel power factor correction (PFC) technique to realize the unity effective power factor and stable output dc voltage for both the distribution systems with sinusoidal or rectangular voltage waveform. Also, this paper presents the estimation and judgment of the input voltage waveform and its stability and the design of the estimation gains. The new control strategy has a notable advantage that it is flexible for both sinusoidal and rectangular waveforms to realize the good characteristics. The effectiveness of the proposed control scheme has been verified by experiments.
\end{abstract}

Keywords: single-phase PFC converter, rectangular-waveform distribution system, estimation and judgment of input voltage waveform, design of gain

\section{Introduction}

A lot of industrial equipment and household electric appliances such as motor drive systems, lighting systems and air conditioners have rectifier circuits for the dc voltage source of the inverter circuits. Diode rectifier circuits with simple configuration are popularly used for the equipment. However, the input current waveform of the diode rectifier circuit is distorted and the harmonic current causes low power factor and harmonic interferences. To avoid this problem, PFC converters have attracted considerable attention ${ }^{(1)}$. However, the system configuration is complicated compared with that of the diode rectifier circuit.

On the other hand, the dc power distribution system has been proposed for the equipment with the inverter circuits ${ }^{(2)}$. However, the isolation and the over-current protection in the dc power distribution system are difficult to realize compared with that in the ac power distribution system. Also, the ac distribution systems with the square-waveform and the trapezoidal-waveform are proposed ${ }^{(3)(4)}$. But the distribution system with the square-waveform in reference (3) cannot be applied to the three-phase system. The system configuration to generate the trapezoidal-waveform is complex. The singlephase or three-phase distribution system with the rectangular voltage waveform has been proposed ${ }^{(5)}$. The distribution system with the rectangular voltage waveform is useful for the equipment with the dc voltage source because the high input power factor is obtained by using the diode rectifier circuits

\footnotetext{
* Nagoya Institute of Technology

Gokiso, Showa, Nagoya 466-8555
}

whose system configuration is simple.

This paper presents a single-phase PFC converter for both the distribution systems with the sinusoidal or rectangular voltage waveform. Also, this paper presents an input voltage estimation because it is necessary to judge between the sinusoidal and rectangular voltage waveforms and to obtain the high input power factor. The voltage estimation algorithm is based on the estimation of the amplitude and phase angle of the input voltage waveform ${ }^{(6)(7)}$. The stability of the proposed estimation algorithm and the design of the estimation gains are shown. Using the proposed estimation algorithm, the conventional PFC converter for the sinusoidal input voltage can also work for the rectangular input voltage. The effectiveness of the proposed estimation and control scheme has been verified by experiments.

\section{Proposed Control Scheme}

2.1 Definition of Sinusoidal and Rectangular Input Voltage The sinusoidal input voltage $e(n-1)$ at the sampling point $n-1$ is given with the effective value $E$ and phase angle $\theta(n-1)$ in (1).

$$
e(n-1)=\sqrt{2} E \cos \theta(n-1)
$$

Figure 1 shows the rectangular input voltage waveform. The relationship between the effective value $E$ and the amplitude $E_{a m p}$ is given in (2).

$$
E=\sqrt{\frac{2}{3}} E_{a m p}
$$

The function $\operatorname{rec} \theta$ is defined as the rectangular waveform of which the amplitude $E_{a m p}$ equals 1 in Fig. $1 . \operatorname{rec} \theta$ is obtained 


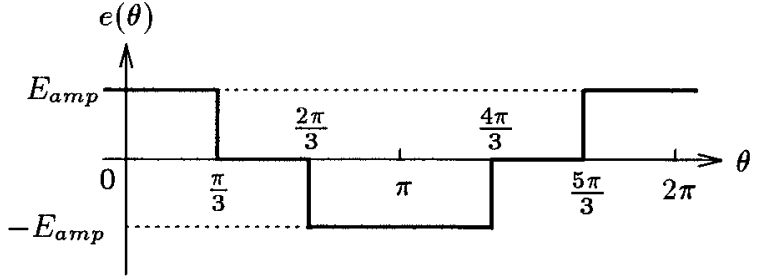

Fig. 1. Rectangular input voltage waveform

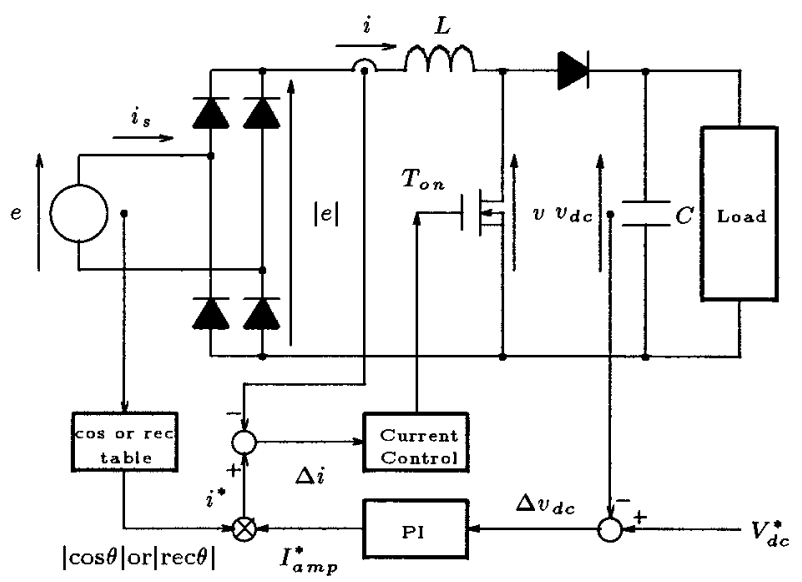

Fig. 2. System configuration of single-phase PFC converter

in (3).

$$
\operatorname{rec} \theta=\left\{\begin{array}{cl}
1 & \left(0 \leq \theta<\frac{\pi}{3}, \frac{5 \pi}{3} \leq \theta<2 \pi\right) \\
0 & \left(\frac{\pi}{3} \leq \theta<\frac{2 \pi}{3}, \frac{4 \pi}{3} \leq \theta<\frac{5 \pi}{3}\right) \\
-1 & \left(\frac{2 \pi}{3} \leq \theta<\frac{4 \pi}{3}\right)
\end{array}\right.
$$

From (2) and (3), the rectangular input voltage $e(n-1)$ at the sampling point $n-1$ is given in (4).

$$
e(n-1)=\sqrt{\frac{3}{2}} \operatorname{Erec} \theta(n-1)
$$

\subsection{Voltage and Current Control ${ }^{(1)}$}

Figure 2 shows the system configuration of the single-phase PFC converter for both sinusoidal and rectangular input voltage waveforms. The control purpose of PFC converter is to realize the unity effective power factor and the stable output dc voltage. To realize the stable output dc voltage, the amplitude of the reactor current reference $I_{a m p}^{*}$ is determined through a PI controller by using the voltage difference $\Delta v_{d c}$ between the dc voltage reference $V_{d c}^{*}$ and the detected output voltage $v_{d c}$. To realize the unity effective power factor, the reactor current reference $i^{*}$ is calculated from the amplitude of the reactor current reference $I_{\text {amp }}^{*}$ and $|\cos \theta|$ or $|\operatorname{rec} \theta|$. Then the on-time of the FET is determined by the current control from the difference $\Delta i$ between the reactor current reference $i^{*}$ and the detected reactor current $i$. In the current control, the estimated input voltage is used to obtain the high frequency response.

2.3 Estimation of Input Voltage Waveform The sampling delay causes the low power factor. In case of the rectangular input voltage, the power factor $P F$ is obtained in (5)

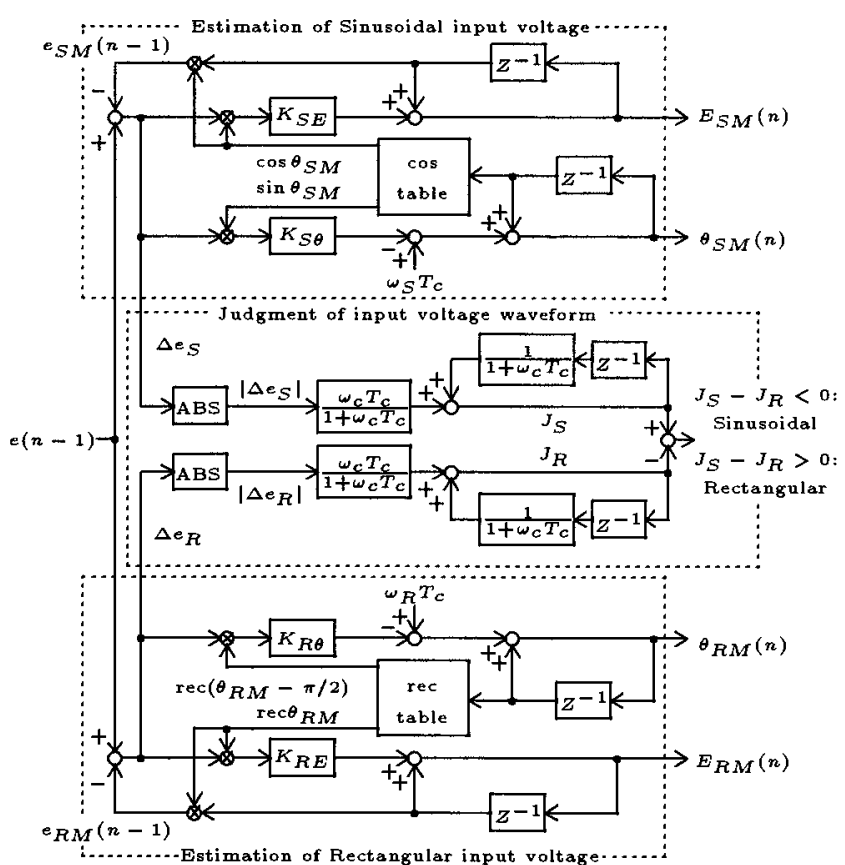

Fig. 3. Block diagram for estimation of input voltage waveform

$$
P F=1-\frac{3}{2 \pi} \Delta \theta \quad-\frac{\pi}{3}<\Delta \theta<\frac{\pi}{3}
$$

where $\Delta \theta$ is the phase difference between the input voltage and current. Under the frequency of $400 \mathrm{~Hz}$ and the sampling delay of $136 \mu \mathrm{s}(\Delta \theta=0.342 \mathrm{rad})$, the power factor $P F$ is 0.837 in (5). To obtain the high input power factor, it is necessary to estimate the input voltage without the sampling delay.

Figure 3 shows the block diagram for the estimation of the input voltage waveform.

The sinusoidal estimated voltage $e_{S M}(n-1)$ and the rectangular estimated voltage $e_{R M}(n-1)$ are given with the estimated amplitude values $E_{S M}$ and $E_{R M}$, and phase angles $\theta_{S M}$ and $\theta_{R M}$ in (6) and (7), respectively.

$$
\begin{aligned}
& e_{S M}(n-1)=E_{S M}(n-1) \cos \theta_{S M}(n-1) \\
& e_{R M}(n-1)=E_{R M}(n-1) \operatorname{rec} \theta_{R M}(n-1)
\end{aligned}
$$

The sinusoidal estimation error of the input voltage, $\Delta e_{S}(n-1)$ can be obtained in (8).

$$
\Delta e_{S}(n-1)=e(n-1)-e_{S M}(n-1)
$$

According to $\Delta e_{S}(n-1)$, the amplitude $E_{S M}(n)$ and the phase angle $\theta_{S M}(n)$ can be estimated in (9) and (10)

$$
\begin{aligned}
E_{S M}(n)= & E_{S M}(n-1) \\
& +K_{S E} \Delta e_{S}(n-1) \cos \theta_{S M} \\
\theta_{S M}(n)= & \theta_{S M}(n-1)+\omega_{S} T_{c} \\
& -K_{S \theta} \Delta e_{S}(n-1) \sin \theta_{S M}
\end{aligned}
$$

where $K_{S E}$ and $K_{S \theta}$ are the gains for the estimation of the amplitude and the phase angle, respectively. $\omega_{S}$ is the angular frequency of the input voltage and $T_{c}$ is the control period.

The rectangular estimation error of the input voltage, $\Delta e_{R}(n-1)$ can be obtained in (11).

$$
\Delta e_{R}(n-1)=e(n-1)-e_{R M}(n-1) .
$$


According to $\Delta e_{R}(n-1)$, the amplitude $E_{R M}(n)$ and the phase angle $\theta_{R M}(n)$ can be estimated in (12) and (13)

$$
\begin{aligned}
E_{R M}(n)= & E_{R M}(n-1) \\
& +K_{R E} \Delta e_{R}(n-1) \operatorname{rec} \theta_{R M} \cdots \cdots \\
\theta_{R M}(n)= & \theta_{R M}(n-1)+\omega_{R} T_{c} \\
& -K_{R \theta} \Delta e_{R}(n-1) \operatorname{rec}\left(\theta_{R M}-\pi / 2\right)
\end{aligned}
$$

where $K_{R E}$ and $K_{R \theta}$ are the gains for the estimation of the amplitude and the phase angle, respectively. $\omega_{R}$ is the angular frequency of the input voltage.

The average absolute errors for the sinusoidal and rectangular waveforms $J_{S}$ and $J_{R}$ are obtained in (14) and (15), respectively

$$
\begin{aligned}
J_{S}(n-1)= & \frac{\omega_{c} T_{c}}{1+\omega_{c} T_{c}}\left|\Delta e_{S}(n-1)\right| \\
& +\frac{1}{1+\omega_{c} T_{c}} J_{S}(n-2) . \\
J_{R}(n-1)= & \frac{\omega_{c} T_{c}}{1+\omega_{c} T_{c}}\left|\Delta e_{R}(n-1)\right| \\
& +\frac{1}{1+\omega_{c} T_{c}} J_{R}(n-2) .
\end{aligned}
$$

where $\omega_{c}$ is the cutoff angular frequency. In the steady-state, $J_{S}$ and $J_{R}$ are compared and then the corresponding estimated voltage waveform of the smaller one is selected as the input voltage waveform. For example, in case $J_{S}<J_{R}$, the sinusoidal input voltage waveform is selected.

\section{Stability and Estimation Gains}

3.1 Stability of Sinusoidal Voltage Estimation Algorithm The sinusoidal estimation errors of the amplitude and the phase angle $\Delta E_{S}(n)$ and $\Delta \theta_{S}(n)$ are defined in (16) and (17), respectively.

$$
\begin{aligned}
& \Delta E_{S}(n)=E_{a m p}-E_{S M}(n) \\
& \Delta \theta_{S}(n)=\theta-\theta_{S M}(n) \cdots
\end{aligned}
$$

The estimation error vector $\boldsymbol{\varepsilon}_{S}$ is defined in (18).

$$
\boldsymbol{\varepsilon}_{S}=\left[\begin{array}{c}
\Delta E_{S} / E_{a m p} \\
\Delta \theta_{S}
\end{array}\right]
$$

The stability of the estimation algorithm can be obtained from the convergence to 0 of $\left|\varepsilon_{S}\right|$. From (1), (6), (8), (16) and (17), the estimation error of the input voltage, $\Delta e_{S}(n-1)$ is obtained by using $\sin \Delta \theta_{S} \simeq \Delta \theta_{S}, \cos \Delta \theta_{S} \simeq 1$ under $\Delta \theta_{S} \simeq 0$ in (19).

$$
\begin{aligned}
\Delta e_{S}(n-1) \simeq & \Delta E_{S}(n-1) \cos \theta_{S M} \\
& -E_{a m p} \Delta \theta_{S}(n-1) \sin \theta_{S M}
\end{aligned}
$$

By using (9), (16) can be rearranged in (20).

$$
\begin{aligned}
\Delta E_{S}(n)= & \Delta E_{S}(n-1) \\
& -K_{S E} \Delta e_{S}(n-1) \cos \theta_{S M}(n-1)
\end{aligned}
$$

By using (10), (17) can be rearranged in (21).

$$
\begin{aligned}
\Delta \theta_{S}(n)= & \Delta \theta_{S}(n-1) \\
& +K_{S \theta} \Delta e_{S}(n-1) \sin \theta_{S M}(n-1)
\end{aligned}
$$

From (19), (20) and (21), the error equation can be obtained in (22)

$$
\boldsymbol{\varepsilon}_{S}(n)=\boldsymbol{A}_{S}\left(\theta_{S M}\right) \boldsymbol{\varepsilon}_{S}(n-1)
$$

where

$$
\begin{aligned}
& \boldsymbol{A}_{S}\left(\theta_{S M}\right) \\
& =\left[\begin{array}{cc}
1-K_{S E} \cos ^{2} \theta_{S M} & K_{S E} \sin \theta_{S M} \cos \theta_{S M} \\
K_{S \theta}^{\prime} \sin \theta_{S M} \cos \theta_{S M} & 1-K_{S \theta}^{\prime} \sin ^{2} \theta_{S M}
\end{array}\right] \\
& K_{S \theta}^{\prime}=E_{a m p} K_{S \theta}
\end{aligned}
$$

$\boldsymbol{A}_{S}\left(\theta_{S M}\right)$ includes the nonlinear functions. By using the following transformation matrix $\boldsymbol{T}_{S}^{-1}$

$$
\boldsymbol{T}_{S}^{-1}=\left[\begin{array}{cc}
\sqrt{\frac{K_{S \theta}^{\prime}}{K_{S E}}} \sin \theta_{S M} & \sqrt{\frac{K_{S E}}{K_{S \theta}^{\prime}}} \cos \theta_{S M} \\
\cos \theta_{S M} & -\sin \theta_{S M}
\end{array}\right], \ldots \ldots
$$

(22) can be diagonalized in (26)

$$
\boldsymbol{\varepsilon}_{S T}(n)=\lambda_{S}\left(\theta_{S M}\right) \boldsymbol{\varepsilon}_{S T}(n-1)
$$

where

$$
\begin{gathered}
\boldsymbol{\varepsilon}_{S T}=\boldsymbol{T}_{S}^{-1} \boldsymbol{\varepsilon}_{S} \ldots \ldots \ldots \ldots \ldots \ldots \ldots \ldots \ldots \ldots \ldots \ldots \ldots \\
\lambda_{S}\left(\theta_{S M}\right)=\boldsymbol{T}_{S}^{-1} \boldsymbol{A}_{S}\left(\theta_{S M}\right) \boldsymbol{T}_{S}=\left[\begin{array}{cc}
\lambda_{S 1} & 0 \\
0 & \lambda_{S 2}
\end{array}\right] \\
\left\{\begin{array}{c}
\lambda_{S 1}=1 \\
\lambda_{S 2}=1-K_{S E} \cos ^{2} \theta_{S M}-K_{S \theta}^{\prime} \sin ^{2} \theta_{S M}
\end{array}\right.
\end{gathered}
$$

$\lambda_{S 1}$ and $\lambda_{S 1}$ are the eigenvalues of $\boldsymbol{A}_{S}\left(\theta_{S M}\right)$.

(1) $K_{S}=K_{S E}=K_{S \theta}^{\prime}$

The eigenvalues in (29) are $\lambda_{S 1}=1, \lambda_{S 2}=1-K_{S}$ and $\lambda_{S}\left(\theta_{S M}\right)$ in (26) can be linear.

$\boldsymbol{T}_{S}^{-1}$ in (25) is the transformation matrix to the $\alpha-\beta$ coordinate system that rotates synchronizing with $\theta_{S M}$. Since the coordinate system rotates synchronizing with $\theta_{S M},\left|\boldsymbol{\varepsilon}_{S}\right|$ converges to 0 under $\lambda_{S 1}=1$ if the absolute value of $\lambda_{S 2}$ is less than 1 . Therefore the stability condition of the gain $K_{S}$ is obtained in (30).

$$
0<K_{S}<2 \quad\left(K_{S}=K_{S E}=K_{S \theta}^{\prime}\right)
$$

(2) $K_{S E} \neq K_{S \theta}^{\prime}$

The eigenvalues $\lambda_{S 1}$ and $\lambda_{S 2}$ in (29) change with $\theta_{S M}$ and $\lambda_{S}\left(\theta_{S M}\right)$ in (26) is nonlinear. Therefore $\left|\boldsymbol{\varepsilon}_{S}\right|$ may not converge to 0 even if the absolute value of $\lambda_{S 2}$ is less than 1 .

From the cases of (1) and (2), the stability of the estimation algorithm can be obtained in (30).

3.2 Stability of Rectangular Voltage Estimation Algorithm The rectangular estimation errors of the amplitude and the phase angle $\Delta E_{R}(n)$ and $\Delta \theta_{R}(n)$ are defined in (31) and (32), respectively.

$$
\begin{aligned}
& \Delta E_{R}(n)=E_{a m p}-E_{R M}(n) \\
& \Delta \theta_{R}(n)=\theta-\theta_{R M}(n) \cdots \cdots
\end{aligned}
$$

The estimation error vector $\boldsymbol{\varepsilon}_{R}$ is defined in (33).

$$
\boldsymbol{\varepsilon}_{R}=\left[\begin{array}{c}
\Delta E_{R} / E_{a m p} \\
\Delta \theta_{R}
\end{array}\right]
$$




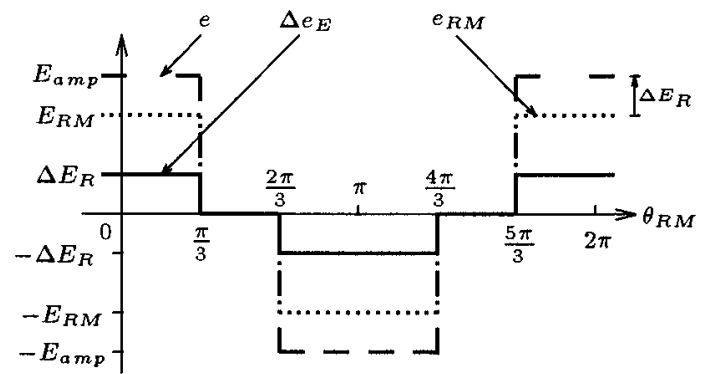

Fig. 4. Input voltage waveforms with amplitude error

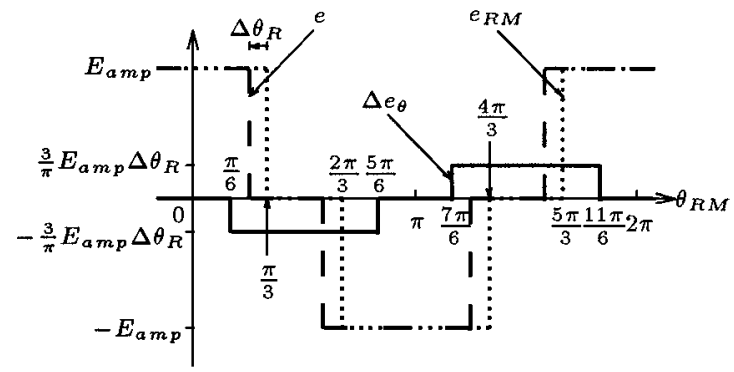

Fig. 5. Input voltage waveforms with phase angle error

The stability of the estimation algorithm can be obtained from the convergence to 0 of $\left|\varepsilon_{R}\right|$. Figure 4 shows the voltage waveforms with the estimation error of the amplitude $\Delta E_{R}$ between the input voltage $e$ and the estimated voltage $e_{R M}$. The estimation error waveform $\Delta e_{E}\left(=e-e_{R M}\right)$ is expressed in (34).

$$
\Delta e_{E}=\Delta E_{R} \operatorname{rec} \theta_{R M}
$$

Figure 5 shows the voltage waveforms with the estimation error of the phase angle $\Delta \theta_{R}$ between the input voltage $e$ and the estimated voltage $e_{R M}$. The estimation error waveform $\Delta e_{\theta}\left(=e-e_{R M}\right)$ is approximated in (35)

$$
\Delta e_{\theta} \simeq-\frac{3}{\pi} E_{\text {amp }} \Delta \theta_{R} \operatorname{rec}\left(\theta_{R M}-\frac{\pi}{2}\right) .
$$

where $\Delta e_{\theta}$ is approximated to obtain the equivalent area of $e-e_{R M}$. The estimation error of the input voltage, $\Delta e_{R}(n-1)$ is the summation of $\Delta e_{E}$ in (34) and $\Delta e_{\theta}$ in (35), and is obtained in (36).

$$
\begin{aligned}
\Delta e_{R}(n-1)= & \Delta e_{E}(n-1)+\Delta e_{\theta}(n-1) \\
\simeq & \Delta E_{R}(n-1) \operatorname{rec} \theta_{R M} \\
& -\frac{3}{\pi} E_{a m p} \Delta \theta_{R}(n-1) \operatorname{rec}\left(\theta_{R M}-\frac{\pi}{2}\right)
\end{aligned}
$$

By using (12), (31) can be rearranged in (37).

$$
\begin{aligned}
\Delta E_{R}(n)= & \Delta E_{R}(n-1) \\
& -K_{R E} \Delta e_{R}(n-1) \operatorname{rec} \theta_{R M}(n-1)
\end{aligned}
$$

\begin{tabular}{|c|c|c|}
\hline phase $\theta_{R M}$ & $A_{R}\left(\theta_{R M}\right)$ & mode \\
\hline $0 \leq \theta_{R M}<\frac{\pi}{6}$ & {$\left[\begin{array}{cc}1-K_{R E} & 0 \\
0 & 1\end{array}\right]$} & 1 \\
\hline$\frac{\pi}{6} \leq \theta_{R M}<\frac{\pi}{3}$ & {$\left[\begin{array}{cc}1-K_{R E} & \frac{3}{\pi} K_{R E} \\
K_{R \theta}^{\prime} & 1-\frac{3}{\pi} K_{R \theta}^{\prime}\end{array}\right]$} & 2 \\
\hline$\frac{\pi}{3} \leq \theta_{R M}<\frac{2 \pi}{3}$ & {$\left[\begin{array}{cc}1 & 0 \\
0 & 1-\frac{3}{\pi} K_{R \theta}^{\prime}\end{array}\right]$} & 3 \\
\hline$\frac{2 \pi}{3} \leq \theta_{R M}<\frac{5 \pi}{6}$ & {$\left[\begin{array}{cc}1-K_{R E} & -\frac{3}{\pi} K_{R E} \\
-K_{R \theta}^{\prime} & 1-\frac{3}{\pi} K_{R \theta}^{\prime}\end{array}\right]$} & 4 \\
\hline$\frac{5 \pi}{6} \leq \theta_{R M}<\frac{7 \pi}{6}$ & {$\left[\begin{array}{cc}1-K_{R E} & 0 \\
0 & 1\end{array}\right]$} & 1 \\
\hline$\frac{7 \pi}{6} \leq \theta_{R M}<\frac{4 \pi}{3}$ & {$\left[\begin{array}{cc}1-K_{R E} & \frac{3}{\pi} K_{R E} \\
K_{R \theta}^{\prime} & 1-\frac{3}{\pi} K_{R \theta}^{\prime}\end{array}\right]$} & 2 \\
\hline$\frac{4 \pi}{3} \leq \theta_{R M}<\frac{5 \pi}{3}$ & {$\left[\begin{array}{cc}1 & 0 \\
0 & 1-\frac{3}{\pi} K_{R \theta}^{\prime}\end{array}\right]$} & 3 \\
\hline$\frac{5 \pi}{3} \leq \theta_{R M}<\frac{11 \pi}{6}$ & {$\left[\begin{array}{cc}1-K_{R E} & -\frac{3}{\pi} K_{R E} \\
-K_{R \theta}^{\prime} & 1-\frac{3}{\pi} K_{R \theta}^{\prime}\end{array}\right]$} & 4 \\
\hline$\frac{11 \pi}{6} \leq \theta_{R M}<2 \pi$ & {$\left[\begin{array}{cc}1-K_{R E} & 0 \\
0 & 1\end{array}\right]$} & 1 \\
\hline
\end{tabular}

By using (13), (32) can be rearranged in (38).

$$
\begin{aligned}
\Delta \theta_{R}(n)= & \Delta \theta_{R}(n-1) \\
& +K_{R \theta} \Delta e_{R}(n-1) \operatorname{rec}\left\{\theta_{R M}(n-1)-\frac{\pi}{2}\right\}
\end{aligned}
$$

Table 1. $A_{R}\left(\theta_{R M}\right)$ for phase $\theta_{R M}$

From (36), (37) and (38), the error equation can be obtained in (39)

$$
\boldsymbol{\varepsilon}_{R}(n)=\boldsymbol{A}_{R}\left(\theta_{R M}\right) \boldsymbol{\varepsilon}_{R}(n-1)
$$

where

$$
\begin{aligned}
& A_{R}\left(\theta_{R M}\right)=\left[\begin{array}{c}
1-K_{R E} \operatorname{rec}^{2} \theta_{R M} \\
K_{R \theta}^{\prime} \operatorname{rec}\left(\theta_{R M}-\frac{\pi}{2}\right) \operatorname{rec} \theta_{R M} \\
\frac{3}{\pi} K_{R E} \operatorname{rec}\left(\theta_{R M}-\frac{\pi}{2}\right) \operatorname{rec} \theta_{R M} \\
1-\frac{3}{\pi} K_{R \theta}^{\prime} \operatorname{rec}^{2}\left(\theta_{R M}-\frac{\pi}{2}\right)
\end{array}\right] \\
& K_{R \theta}^{\prime}=E_{a m p} K_{R \theta} \ldots \ldots \ldots \ldots \ldots \ldots \ldots \ldots \ldots
\end{aligned}
$$

Table 1 shows $\boldsymbol{A}_{R}\left(\theta_{R M}\right)$ for the estimated phase angle $\theta_{R M}$. $\boldsymbol{A}_{R}\left(\theta_{R M}\right)$ has 4 modes.

(1) Mode 1

The eigenvalues of $\boldsymbol{A}_{R}\left(\theta_{R M}\right)$ are obtained in (42).

$$
\left.\begin{array}{l}
\lambda_{R 11}=1-K_{R E} \\
\lambda_{R 12}=1
\end{array}\right\}
$$

The condition that the absolute value of $\lambda_{R 11}$ is less than 1 is obtained in (43).

$$
0<K_{R E}<2
$$

(2) Mode 2

The eigenvalues of $\boldsymbol{A}_{R}\left(\theta_{R M}\right)$ are obtained in (44).

$$
\left.\begin{array}{l}
\lambda_{R 21}=1 \\
\lambda_{R 22}=1-K_{R E}-\frac{3}{\pi} K_{R \theta}^{\prime}
\end{array}\right\}
$$

The condition that the absolute value of $\lambda_{R 22}$ is less than 1 is obtained in (45).

$$
-\frac{\pi}{3} K_{R E}<K_{R \theta}^{\prime}<-\frac{\pi}{3} K_{R E}+\frac{2}{3} \pi .
$$

(3) Mode 3

The eigenvalues of $\boldsymbol{A}_{R}\left(\theta_{R M}\right)$ are obtained in (46).

$$
\left.\begin{array}{l}
\lambda_{R 31}=1 \\
\lambda_{R 32}=1-\frac{3}{\pi} K_{R \theta}^{\prime}
\end{array}\right\}
$$

The condition that the absolute value of $\lambda_{R 32}$ is less than 1 is obtained in (47). 


$$
0<K_{R \theta}^{\prime}<\frac{2}{3} \pi
$$

(4) Mode 4

The eigenvalues of $\boldsymbol{A}_{R}\left(\theta_{R M}\right)$ are obtained in (48).

$$
\left.\begin{array}{l}
\lambda_{R 41}=1 \\
\lambda_{R 42}=1-K_{R E}-\frac{3}{\pi} K_{R \theta}^{\prime}
\end{array}\right\}
$$

The condition that the absolute value of $\lambda_{R 42}$ is less than 1 is obtained in (49).

$$
-\frac{\pi}{3} K_{R E}<K_{R \theta}^{\prime}<-\frac{\pi}{3} K_{R E}+\frac{2}{3} \pi
$$

Since the coordinate system changes with $\theta_{R M},\left|\boldsymbol{\varepsilon}_{R}\right|$ converges to 0 under $\lambda_{R 12}=\lambda_{R 21}=\lambda_{R 31}=\lambda_{R 41}=1$ if the absolute values of $\lambda_{R 11}, \lambda_{R 22}, \lambda_{R 32}$ and $\lambda_{R 42}$ are less than 1 . Therefore, from (43), (45), (47) and (49), the stability condition of the gain $K_{R E}$ and $K_{R \theta}^{\prime}$ is obtained in (50).

$$
\left.\begin{array}{l}
0<K_{R E} \\
0<K_{R \theta}^{\prime} \\
K_{R \theta}^{\prime}<-\frac{\pi}{3} K_{R E}+\frac{2}{3} \pi
\end{array}\right\}
$$

\subsection{Design of Gains for Sinusoidal Voltage Estimation}

$\boldsymbol{A}_{S}\left(\theta_{S M}\right)$ in (23) consists of the definite matrix $\boldsymbol{A}_{S 0}$ and the nonlinear matrix $\boldsymbol{A}_{S 1}$ as follows.

$$
\begin{aligned}
& \boldsymbol{A}_{S}\left(\theta_{S M}\right)=\boldsymbol{A}_{S 0}+\boldsymbol{A}_{S 1} \ldots \ldots \ldots \ldots \ldots \\
& \boldsymbol{A}_{S 0}=\left[\begin{array}{cc}
1-\frac{K_{S}}{2} & 0 \\
0 & 1-\frac{K_{S}}{2}
\end{array}\right] \ldots \ldots \ldots \ldots \\
& \boldsymbol{A}_{S 1}=\frac{K_{S}}{2}\left[\begin{array}{cc}
-\cos 2 \theta_{S M} & \sin 2 \theta_{S M} \\
\sin 2 \theta_{S M} & \cos 2 \theta_{S M}
\end{array}\right]
\end{aligned}
$$

$\boldsymbol{A}_{S 1}$ in (53) oscillates with twice the frequency of the input voltage and its average value is 0 . Therefore $\boldsymbol{A}_{S}\left(\theta_{S M}\right)$ can be approximated in (54) if the convergence characteristic of the input voltage estimation is slow enough compared with twice the frequency of the input voltage

$$
\begin{aligned}
\boldsymbol{\varepsilon}_{S}(n) & =\left(\boldsymbol{A}_{S 0}+\boldsymbol{A}_{S 1}\right) \boldsymbol{\varepsilon}_{S}(n-1) \\
& \simeq \boldsymbol{A}_{S 0} \boldsymbol{\varepsilon}_{S}(n-1) \\
& =\left(1-\frac{K_{S}}{2}\right)^{n} \boldsymbol{\varepsilon}_{S}(0) \ldots \ldots
\end{aligned}
$$

where $\varepsilon_{S}(0)=\left[\Delta E_{S}(0) / E_{a m p} \Delta \theta_{S}(0)\right]^{T}$ is the initial value of the estimation error vector. From the equivalence of the response for the sampling point $n$ in (54) and the time response for the first order system with time constant $\tau_{S}$, the relationship between the eigenvalue $1-K_{S} / 2$ and time constant $\tau_{S}$ is given in (55).

$$
1-\frac{K_{S}}{2}=e^{-\frac{T_{c}}{\tau_{S}}}
$$

From (24) and (55), the gains for input voltage estimation, $K_{S E}$ and $K_{S \theta}$ can be determined in (56).

$$
\left.\begin{array}{l}
K_{S E}=2\left(1-e^{-\frac{T_{c}}{\tau_{S}}}\right) \\
K_{S \theta}=\frac{2}{E_{a m p}}\left(1-e^{-\frac{T_{c}}{\tau_{S}}}\right)
\end{array}\right\} .
$$

\subsection{Design of Gains for Rectangular Voltage Estima-} tion $\boldsymbol{A}_{R}\left(\theta_{R M}\right)$ in (40) consists of the definite matrix $\boldsymbol{A}_{R 0}$ and the nonlinear matrix $\boldsymbol{A}_{R 1}$ as follows.

$$
\begin{gathered}
\boldsymbol{A}_{R}\left(\theta_{R M}\right)=\boldsymbol{A}_{R 0}+\boldsymbol{A}_{R 1} \ldots \ldots \ldots \ldots \ldots \ldots \ldots \ldots \ldots \ldots \\
\boldsymbol{A}_{R 0}=\left[\begin{array}{cc}
1-\frac{2}{3} K_{R E} & 0 \\
0 & 1-\frac{2}{\pi} K_{R \theta}^{\prime}
\end{array}\right] \ldots \ldots \ldots \ldots \ldots \\
\boldsymbol{A}_{R 1}=\left[\begin{array}{c}
-K_{R E}\left(\operatorname{rec}^{2} \theta_{R M}-\frac{2}{3}\right) \\
K_{R \theta}^{\prime} \operatorname{rec}\left(\theta_{R M}-\frac{\pi}{2}\right) \operatorname{rec} \theta_{R M} \\
\frac{3}{\pi} K_{R E} \operatorname{rec}\left(\theta_{R M}-\frac{\pi}{2}\right) \operatorname{rec} \theta_{R M} \\
* \quad-\frac{3}{\pi} K_{R \theta}^{\prime}\left\{\operatorname{rec}^{2}\left(\theta_{R M}-\frac{\pi}{2}\right)-\frac{2}{3}\right\}
\end{array}\right] \ldots \ldots .(5)
\end{gathered}
$$

$\boldsymbol{A}_{R 1}$ in (59) oscillates with twice the frequency of the input voltage and its average value is 0 . Therefore, $\boldsymbol{A}_{R}\left(\theta_{R M}\right)$ can be approximated in (60) if the convergence characteristic of the input voltage estimation is slow enough compared with twice the frequency of the input voltage

$$
\begin{aligned}
\boldsymbol{\varepsilon}_{R}(n) & =\left(\boldsymbol{A}_{R 0}+\boldsymbol{A}_{R 1}\right) \boldsymbol{\varepsilon}_{R}(n-1) \\
& \simeq \boldsymbol{A}_{R 0} \boldsymbol{\varepsilon}_{R}(n-1) \\
& =\left[\begin{array}{cc}
1-\frac{2}{3} K_{R E} & 0 \\
0 & 1-\frac{2}{\pi} K_{R \theta}^{\prime}
\end{array}\right]^{n} \boldsymbol{\varepsilon}_{R}(0)
\end{aligned}
$$

where $\varepsilon_{R}(0)=\left[\Delta E_{R}(0) / E_{a m p} \Delta \theta_{R}(0)\right]^{T}$ is the initial value of the estimation error vector. From the equivalence of the response for the sampling point $n$ in (60) and the time response for the first order system with time constant $\tau_{R}$, the relationship between the eigenvalue $1-2 K_{R E} / 3,1-2 K_{R \theta}^{\prime} / \pi$ and time constant $\tau_{R}$ is given in (61).

$$
\left.\begin{array}{l}
1-\frac{2}{3} K_{R E}=e^{-\frac{T_{c}}{\tau_{R}}} \\
1-\frac{2}{\pi} K_{R \theta}^{\prime}=e^{-\frac{T_{c}}{\tau_{R}}}
\end{array}\right\}
$$

From (41) and (61), the gains for input voltage estimation, $K_{R E}$ and $K_{R \theta}$ can be determined in (62).

$$
\left.\begin{array}{l}
K_{R E}=\frac{3}{2}\left(1-e^{-\frac{T_{c}}{\tau_{R}}}\right) \\
K_{R \theta}=\frac{\pi}{2 E_{a m p}}\left(1-e^{-\frac{T_{c}}{\tau_{R}}}\right)
\end{array}\right\}
$$

\section{Experimental Results}

4.1 System Configuration Figure 6 shows a $600 \mathrm{~W}$ single-phase PFC converter for both sinusoidal and rectangular input voltage waveforms for experiments. The specifications are shown in Table 2. A DSP (TMS320C50) is used for the controller of the PFC converter.

Table 3 shows the gains for the input voltage estimation. The gains are designed as the time constant $\tau_{S}=\tau_{R}=3.89$ $\mathrm{ms}$ in (56) and (62).

4.2 Sinusoidal Voltage Estimation Figure 7 shows the theoretical and experimental convergence characteristics for the amplitude disturbances of $\pm 30 \mathrm{~V}$ at $\theta=0 \mathrm{deg}$ under the sinusoidal voltage estimation. Figure 8 shows the theoretical and experimental convergence characteristics for the phase angle disturbance of $\pm 30 \mathrm{deg}$ at $\theta=0 \mathrm{deg}$. The dotted lines are the theoretical characteristics in (54), the solid lines are the experimental ones. From Figs. 7 and 8, the experimental convergence characteristics are close to the theoretical ones. 


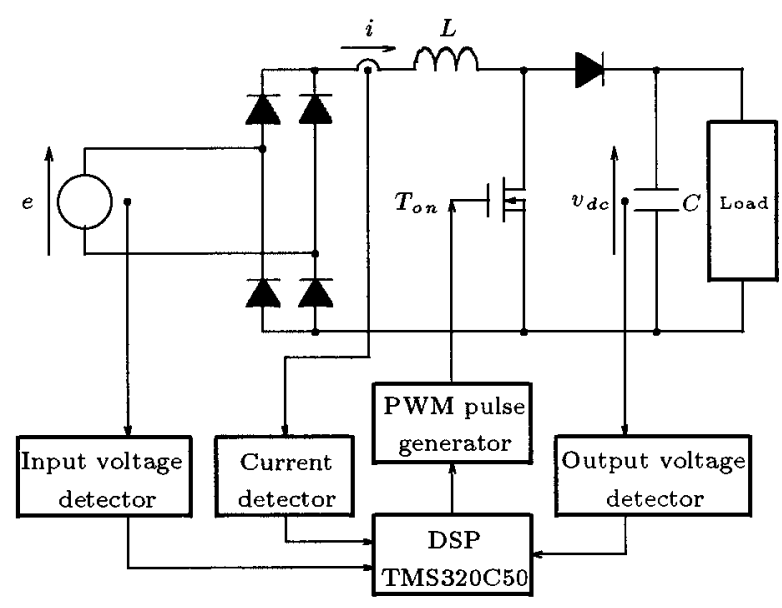

Fig. 6. Hardware configuration

Table 2. System specifications

\begin{tabular}{c|c}
\hline Rated output & $600 \mathrm{~W}$ \\
Voltage source $e$ & AC200 V, $60 \mathrm{~Hz}$, Sinusoidal \\
& or \\
& AC200 V, $400 \mathrm{~Hz}$, Rectangular \\
Rated output voltage & $\mathrm{DC} 380 \mathrm{~V}$ \\
Inductance $L$ & $1.72 \mathrm{mH}$ \\
Smoothing capacitance $C$ & $1500 \mu \mathrm{F}$ \\
Switching frequency & $58.8 \mathrm{kHz}$ \\
Control period $T_{c}$ & $68 \mu \mathrm{s}$ \\
\hline
\end{tabular}

Table 3. Experimental conditions

\begin{tabular}{|c|c|}
\hline $\begin{array}{l}\text { Estimation of } \\
\text { sinusoidal } \\
\text { input voltage }\end{array}$ & $\begin{array}{c}\text { Gain for estimation of amplitude } \\
K_{S E}=0.0347 \\
\text { Gain for estimation of phase angle } \\
K_{S \theta}=0.000123 \mathrm{rad} / \mathrm{V}\end{array}$ \\
\hline $\begin{array}{l}\text { Estimation of } \\
\text { rectangular } \\
\text { input voltage }\end{array}$ & $\begin{array}{c}\text { Gain for estimation of amplitude } \\
K_{R E}=0.0260 \\
\text { Gain for estimation of phase angle } \\
K_{R \theta}=0.000111 \mathrm{rad} / \mathrm{V}\end{array}$ \\
\hline Voltage control & $\begin{array}{c}\text { Natural angular frequency } \omega_{n}=2 \pi \times 1 \mathrm{rad} / \mathrm{s} \\
\text { Dumping ratio } \zeta=1\end{array}$ \\
\hline $\begin{array}{l}\text { Current control for } \\
\text { sinusoidal } \\
\text { input voltage }\end{array}$ & $\begin{array}{c}\text { Natural angular frequency } \omega_{n}=2 \pi \times 600 \mathrm{rad} / \mathrm{s} \\
\text { Dumping ratio } \zeta=1\end{array}$ \\
\hline $\begin{array}{l}\text { Current control for } \\
\text { rectangular } \\
\text { input voltage }\end{array}$ & $\begin{array}{c}\text { Natural angular frequency } \omega_{n}=2 \pi \times 100 \mathrm{rad} / \mathrm{s} \\
\text { Dumping ratio } \zeta=1\end{array}$ \\
\hline
\end{tabular}

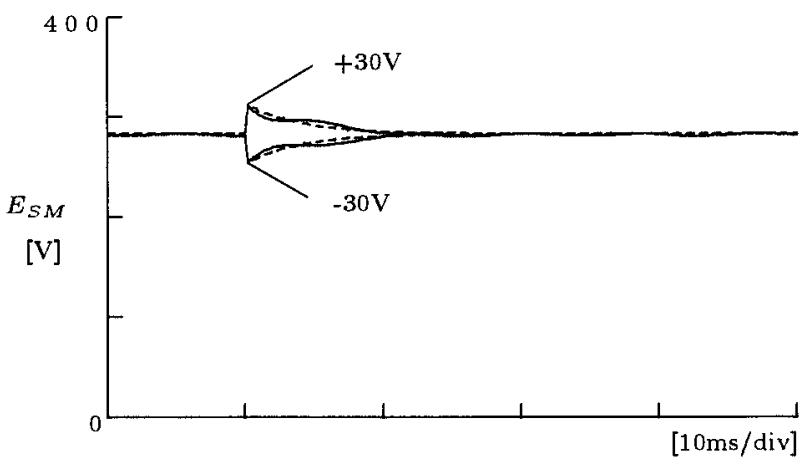

Fig. 7. Theoretical and Experimental Convergence characteristics for amplitude disturbances under sinusoidal voltage estimation

Figure 9 shows the convergence characteristics for the amplitude disturbances of $-300 \sim+300 \mathrm{~V}$ at $\theta=0 \mathrm{deg}$. Figure 10 shows the convergence characteristics for the phase

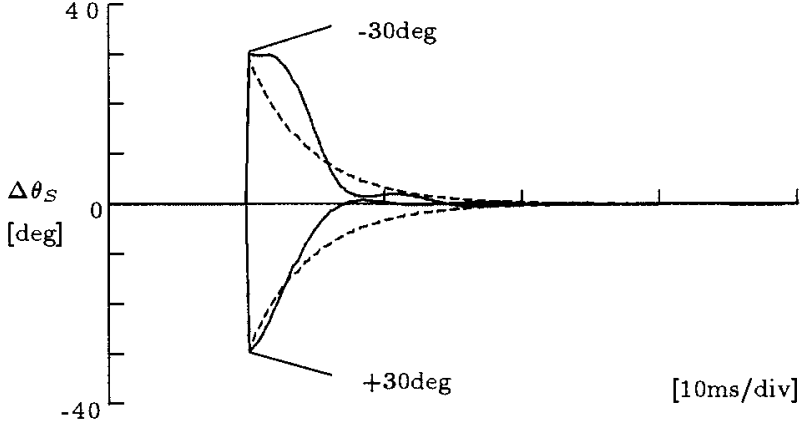

Fig. 8. Theoretical and Experimental Convergence characteristics for phase angle disturbances under sinusoidal voltage estimation

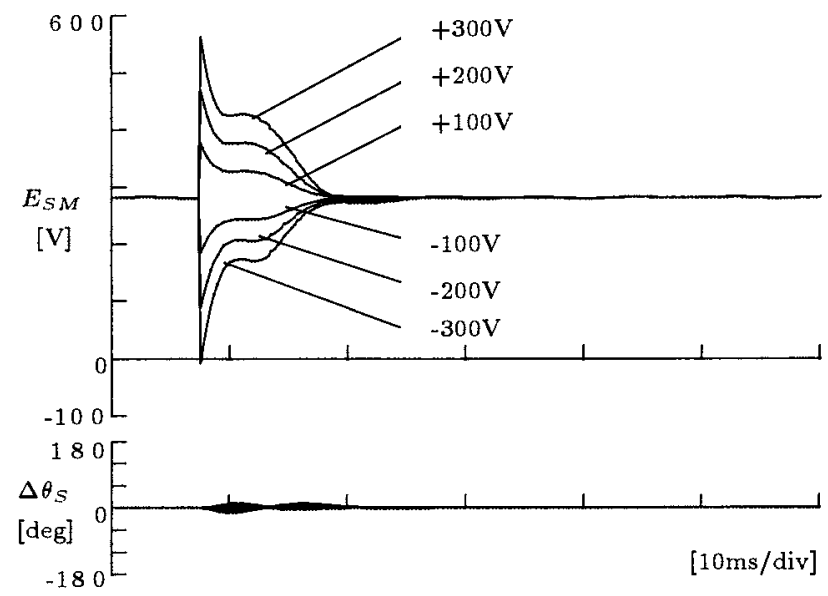

Fig. 9. Convergence characteristics for amplitude disturbances under sinusoidal voltage estimation

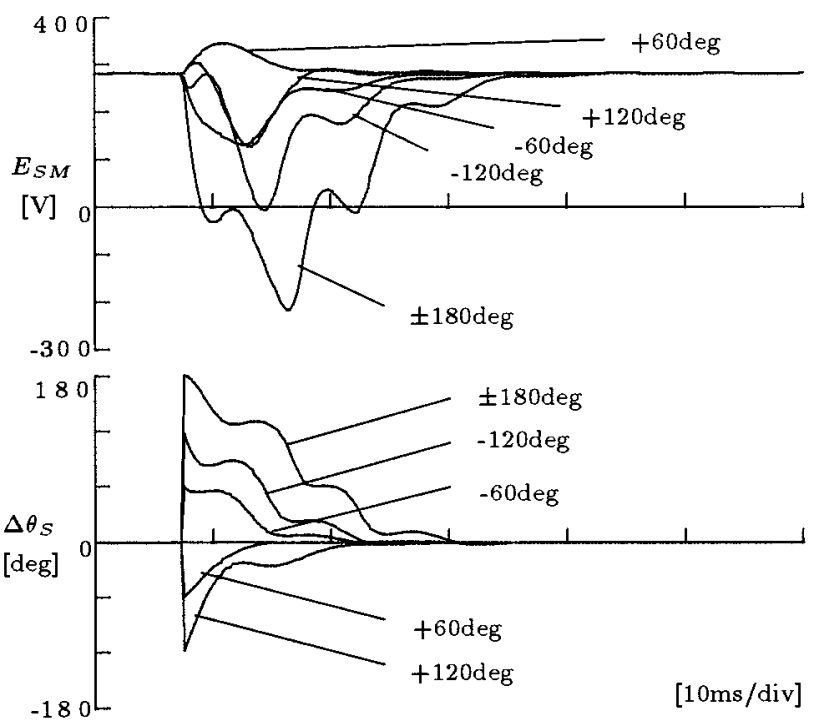

Fig. 10. Convergence characteristics for phase angle disturbances under sinusoidal voltage estimation

angle disturbances of $-180 \sim+180 \mathrm{deg}$ at $\theta=0 \mathrm{deg}$. From Fig. 9, the disturbances of the amplitude do not affect the estimation errors of the phase angle $\Delta \theta_{S}$. From Fig. 10, the estimated amplitude $E_{S M}$ decreases transiently for the disturbances of the estimated phase angle. Then it converges to the amplitude $E_{a m p}$ as the estimation error of the phase angle $\Delta \theta_{S}$ converges to 0 . 


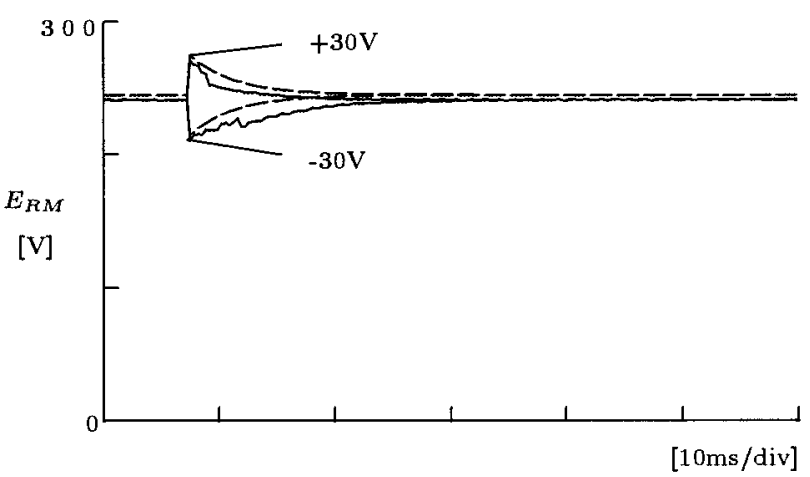

Fig. 11. Theoretical and Experimental Convergence characteristics for amplitude disturbances under rectangular voltage estimation

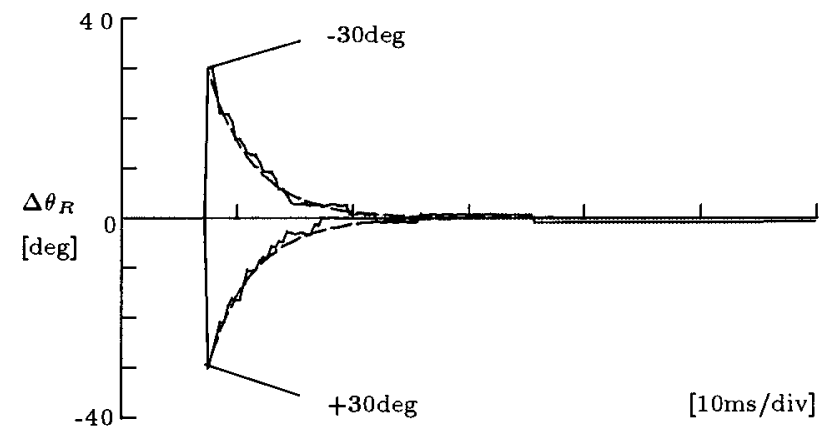

Fig. 12. Theoretical and Experimental Convergence characteristics for phase angle disturbances under rectangular voltage estimation

As above, the sinusoidal voltage estimation algorithm can converge for the disturbances of the amplitude of $-300 \sim$ $+300 \mathrm{~V}$ and the phase angle of $-180 \sim+180 \mathrm{deg}$ within $40 \mathrm{~ms}$ of the input voltage.

4.3 Rectangular Voltage Estimation Figure 11 shows the theoretical and experimental convergence characteristics for the amplitude disturbances of $\pm 30 \mathrm{~V}$ at $\theta=0 \mathrm{deg}$ under the rectangular voltage estimation. Figure 12 shows the theoretical and experimental convergence characteristics for the phase angle disturbances of $\pm 30 \mathrm{deg}$ at $\theta=0 \mathrm{deg}$. The dotted lines are the theoretical characteristics in (60), the solid lines are the experimental ones. From Fig. 11 and 12, however the estimation errors of the amplitude and phase angle are respectively about $5 \mathrm{~V}$ and $1 \mathrm{deg}$ in the maximum, the average experimental convergence characteristics are close to the theoretical ones. The reason of the steady-state errors is that the input voltage waveform is not ideal and the estimation error is always caused.

Figure 13 shows the convergence characteristics for the amplitude disturbances of $-250 \sim+250 \mathrm{~V}$ at $\theta=0 \mathrm{deg}$. Figure 14 shows the convergence characteristics for the phase angle disturbances of $-180 \sim+180 \mathrm{deg}$ at $\theta=0 \mathrm{deg}$. From Fig. 13 and 14, the rectangular voltage estimation algorithm can respectively converge $\Delta E_{R}$ and $\Delta \theta_{R}$ to $0 \sim+5 \mathrm{~V}$ and $-1 \sim+1$ deg for the disturbances of the amplitude of $-250 \sim$ $+250 \mathrm{~V}$ and the phase angle of $-180 \sim+180 \mathrm{deg}$ within 40 $\mathrm{ms}$ of the input voltage.

4.4 Control Characteristics Figures 15 and 16 show the experimental results of the proposed control scheme using the sinusoidal and rectangular input voltages under the

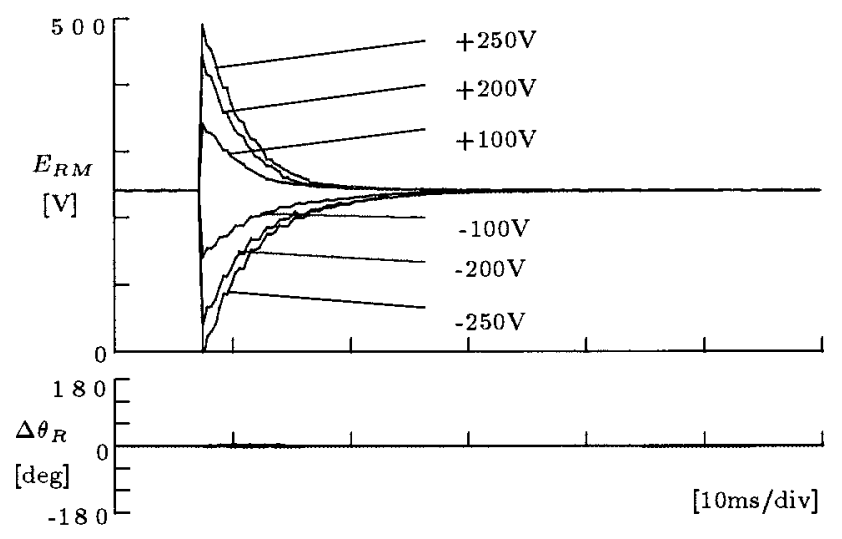

Fig. 13. Convergence characteristics for amplitude disturbances under rectangular voltage estimation

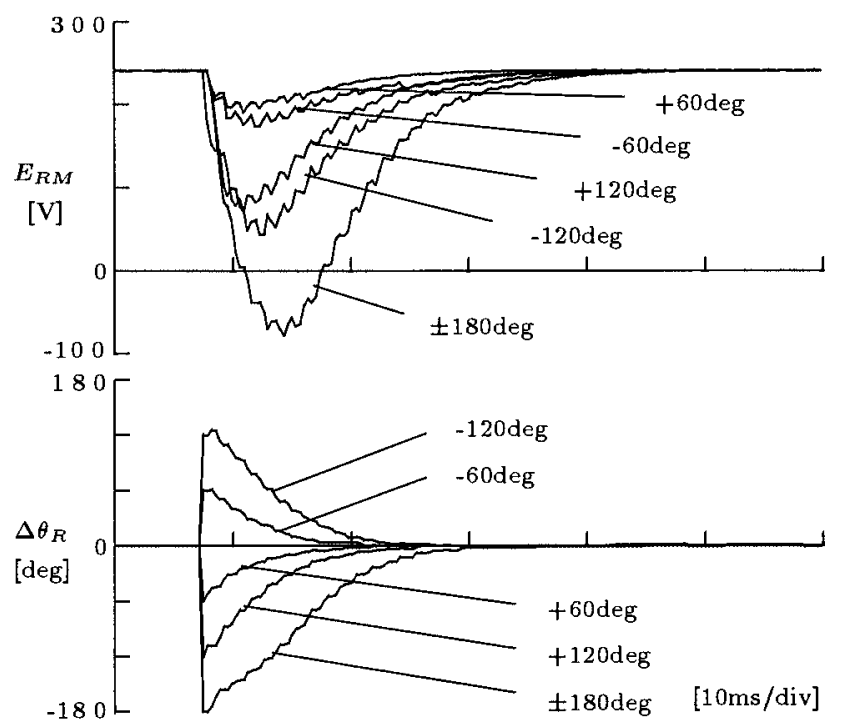

Fig. 14. Convergence characteristics for phase angle disturbances under rectangular voltage estimation

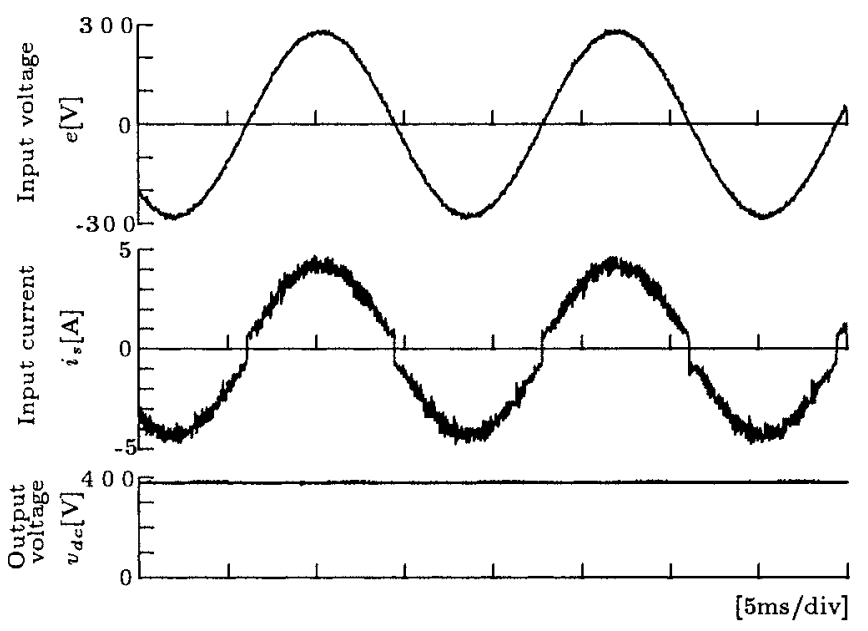

Fig. 15. Experimental results for sinusoidal input voltage

rated load, respectively. The power factor for the sinusoidal and rectangular input voltages, measured by using the Digital Power Meter (WT1030, YOKOGAWA), are 0.995 and 0.949, respectively. Although there are some estimation errors of the rectangular waveform from Fig. 11 to Fig. 14 because of the unideal input voltage, the good characteristics of the PFC 


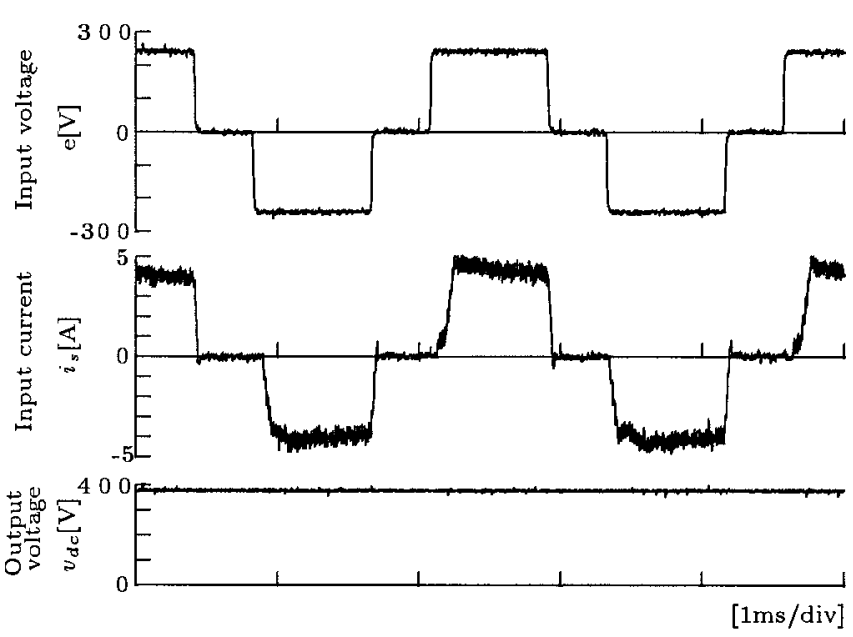

Fig. 16. Experimental results for rectangular input voltage

converter system are obtained. In Fig. 16 there are some current overshoot when the rectangular input current increases and decreases from 0 because of the discontinuous conduction mode of the reactor current.

\section{Conclusions}

This paper presents the input voltage estimation of the single-phase PFC converter for both the sinusoidal and rectangular input voltages. The stability of the estimation algorithm and the design of gains for the estimation are shown.

Using the proposed input voltage estimation algorithm, the estimation errors converge within $40 \mathrm{~ms}$ of the input voltage under the amplitude and phase angle disturbance. The power factors are 0.995 and 0.949 when the sinusoidal and rectangular waveforms are used as the input voltages, respectively.

(Manuscript received May 10, 2005,

revised Oct. 3, 2005)

This paper was presented at IPEC-Niigata 2005, and approved for publication in the IEEJ Transactions on Industry Applications Society.

\section{References}

( 1 ) S. Amano, T. Takeshita, and N. Matsui: "Suppression of Low Order Harmonic Currents for Single-Phase PFC Converter", Proceedings of the 2000 Japan Industry Applications Society Conference, Vol.1, pp.481-484 (2000)

( 2 ) R. Zhang, F.C. Lee, D. Boroyevich, C. Liu, and L. Chen: "AC Load Conditioner and DC Bus Conditioner for a DC Distribution Power System", Proceedings of the 2000 IEEE 31st Annual Power Electronics Specialists Conference, Vol.1, pp.107-112 (2000)

( 3 ) S. Kondo and H. Meguro: "Feasibility Study on Square-wave-voltage power Distribution System", IEEJ Trans. Industy Appl., Vol.116-D, No.5, pp.556562 (1996-5)

( 4 ) Y. Sato and T. Noguchi: "Trapezoidal-Wave-Voltage Power Distribution System with UPS Function", Proceedings of the 1999 Japan Industy Applications Society Conference, Vol.1, pp.219-222 (1999)
( 5 ) W. Sugiyama, S. Watanabe, and T. Takeshita: "Configuration and Performance of Three-Phase Rectangular-Wave Distribution System", The Papers of Joint Technical Meeting on Semiconductor Power Converter and Industry Electric and Electronic, pp.55-60 (2004)

( 6 ) T. Takeshita and N. Matsui: "Single-Phase High Power Factor PWM Converter without Source Phase Angle and Voltage Detectors", IEEJ Trans. Industy Appl., Vol.113-D, No.10, pp.1209-1215 (1993-10)

( 7 ) K. Toyama, T. Takeshita, and N. Matsui: "Stability and Initial Estimation of Power Source Voltage Sensorless Single-Phase PWM AC/DC Converter", IEEJ Trans. Industy Appl., Vol.116-D, No.3, pp.354-360 (1996-3)

Hiroatu Fukuoka (Member) was born in Aichi, Japan, on November

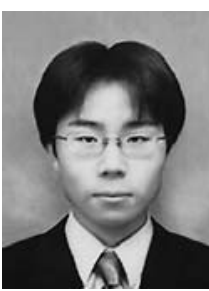
4, 1979. He received the B.S. and M.S. degrees in electrical engineering from Nagoya Institute of Technology, Nagoya, Japan, in 2003, 2005 respectively. He was engaged in research on power converter system. He is currently working in Mitsubishi Electric Corporation.

Quan Li (Non-member) was born in China, on June 15, 1980. He re-

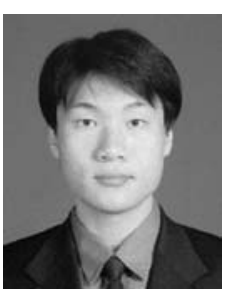
ceived the B.S. degree in electrical engineering from Tsinghua University, Beijing, China, in 2003. He is currently a Master Course student of the same college. He is engaged in research on power converter system.

Toshiyuki Kouno (Student Member) was born in Gifu, Japan, on May

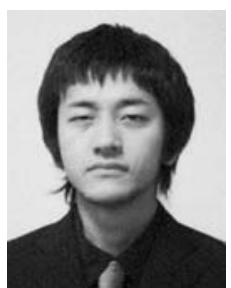
14, 1982. He received the B.S. degree in electrical engineering from Nagoya Institute of Technology, Nagoya, Japan, in 2005. He is currently a Master Course student of the same college. He is engaged in research on power converter system.

Takaharu Takeshita (Member) was born in Aichi, Japan, on August

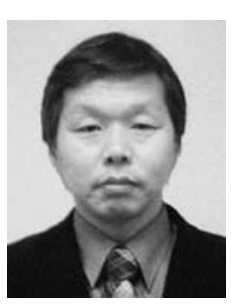
23, 1959. He received the B.S. and M.S. degrees in electrical engineering from Nagoya Institute of Technology, Nagoya Japan, in 1982 and 1984, respectively, and the Ph.D. degree from Nagoya University, Nagoya, Japan, in 1990. Since 1991, he has been with Nagoya Institute of Technology, where he is currently a full professor and is engaged in research on power converter system and motor control. He is a member of the Society of Instrument and Control Engineers,

and IEEE. 